\section{VAT free PPE}

The Government has announced that PPE equipment purchased between 1 May and 31 July 2020 will be VAT free.

Trycare supply an extensive range of PPE products to meet the individual requirements of every member of the practice team. This includes an extensive range of disposable drapes and gowns.

Trycare's disposable sterile surgical gowns and patient drapes are made of a special man-made material called SMS, which is a fluid repellent breathable material to ensure the comfort of the wearer during the operative procedure.

SMS stands for spunbond/meltblown/ spunbond and is a special material which does not have a polythene layer as this would make it too warm to wear for long periods.

Trycare offer their drapes, gowns and ancillary items individually, in bulk packs and in a variety of off-the-shelf packs designed to meet the requirements of different clinical procedures. The latter include their most popular Surgikit as well as their Surgitwo, Endokit, Minimal Implant Kit, Dental Apicectomy Kit, Universal Drape Kit and Economy Drape Kit. For details of the contents and prices visit the Trycare website www.trycare.co.uk or page 61 of their 2019 Surgical Essentials Catalogue.

Should none of the above contain exactly what you want then Trycare can supply customised drape kits designed to meet the individual needs of the practice.

For more information about Trycare's comprehensive VAT free PPE range, including drapes and gowns, contact your local Trycare Representative, call 01274 885544 or visit www.trycare.co.uk.

\section{All your patients covered}

Wisdom Toothbrushes has more than 235 years of experience in the oral healthcare market. As such, it offers a comprehensive range of oral hygiene products to meet the interdental cleaning needs of all your patients.

Have you tried...

- The innovative Wisdom Clean Between Pro Rubber Interdental Brushes

- Wisdom Pro-flex Wire Interdental Brushes

- Wisdom Clean Between Easy Slide Tensioning Flossers with PTFE tape.
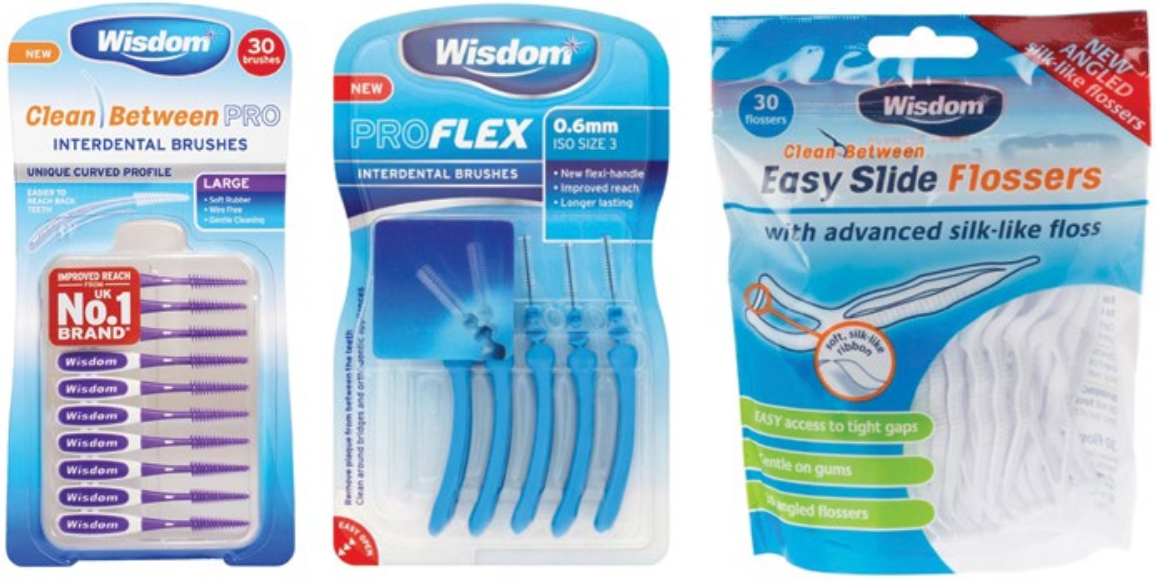

\section{Growth summit to be held} online

Align Technology, Inc. has announced it is moving its originally scheduled Dubrovnik Growth Summit to an exciting new virtual platform. The Summit will combine the latest technologies in hyper-connected broadcasting, and will feature a TV studio with a news anchor moderating the event - to beam some of the brightest minds in dentistry and business directly into dentists' homes.

This is not a webinar, but a full two-day symposium, broadcast on 19 and 20 June, which has been curated for dentists - whether they are current Invisalign providers - or aspire to be - to look beyond the current situation and learn how to prepare for a life back in practice. Joining this unique digital experience will present an opportunity to connect with thought leaders and absorb the unrivaled clinical, business development and personal growth modules which Align Technology has curated.

The progressive programme covers many in-demand topics from leadership and influence, to crisis management, patient connectivity and communication, recovery and business prosperity, comprehensive and multidisciplinary dentistry, diagnoses, treatment planning and protocols, facial driven treatment planning, ways to lead with digital (iTero), and personal growth and wellbeing.

The impressive line up to help the profession in these challenging times includes Dr Ingo Baresel, Dr Karla Soto, Dr David Galler and Dr Raman Aulakh.

The Growth Summit will also be hosting a diverse range of speakers to talk about non-dental topics which are impacting the world of dentistry, including personal and business growth, marketing and empowerment.

Now is the perfect time for dentists to reflect on their skills and their businesses, and what the new consumer will want when practice doors reopen.

For more details about Align Technology's 2020 European Growth Summit on 19-20 June 2020, and how to sign up to participate, visit: http:// aligngrowthsummit.com/. 